Just, Thomas; Stoor, Susann; Klefenz, Frank; Husar, Peter:

\title{
Spike sorting algorithm for multichannel MEA recordings based on cross correlation
}

Zuerst erschienen in: Biomedical Engineering = Biomedizinische Technik. - Berlin [u.a.] : de Gruyter. - 58 (2013), Suppl. 1, Track-G, art. [4198], 2 pp.

Erstveröffentlichung: $\quad$ 2013-09-07

ISSN (online): $\quad$ 1862-278X

ISSN (print): 0013-5585

DOI: $\quad 10.1515 /$ bmt-2013-4198

[Zuletzt gesehen: 2019-07-18]

„Im Rahmen der hochschulweiten Open-Access-Strategie für die Zweitveröffentlichung identifiziert durch die Universitätsbibliothek IImenau."

"Within the academic Open Access Strategy identified for deposition by Ilmenau University Library."

„Dieser Beitrag ist mit Zustimmung des Rechteinhabers aufgrund einer (DFGgeförderten) Allianz- bzw. Nationallizenz frei zugänglich."

"This publication is with permission of the rights owner freely accessible due to an Alliance licence and a national licence (funded by the DFG, German

Research Foundation) respectively." 


\title{
Spike sorting algorithm for multichannel MEA recordings based on cross correlation
}

\author{
Thomas Just ${ }^{1}$, Susann Stoor ${ }^{1}$, Frank Klefenz ${ }^{2}$, Peter Husar ${ }^{1}$ \\ ${ }^{1}$ Institute of Biomedical Engineering and Informatics, Ilmenau University of Technology, Germany \\ ${ }^{2}$ Bio Inspired Computing Group, Fraunhofer Institute for Digital Media Technology, Germany \\ thomas.just@tu-ilmenau.de
}

\begin{abstract}
For evaluation of extra-cellular recordings with multi-electrode arrays (MEAs) an algorithm is needed to detect different types of cellular activity. The algorithm presented in this paper is based on correlation. It is an efficient and simple algorithm which is compared with the wave_clus toolbox. In contrast this toolbox is using wavelets for clustering the cell activity.
\end{abstract}

Keywords: clustering, correlation, multi electrode array, spike sorting

\section{Introduction}

When attempting to understand complex neuronal networks the use of multi electrode arrays is necessary. For evaluation of the recordings of neuronal activities, the detection of spontaneous or stimulated activity is needed. Active cells are producing spikes and group of spikes which can be analyzed by e.g. statistic methods [1]. The wave clus toolbox uses spike detection by feature extraction with wavelet coefficients and superparamagnetic clustering into classes [2]. The spikes are classified by their time curve. This procedure is limited by the number of used wavelet coefficients. The maximum number is 45, so more coefficients could separate probably more classes. For this purpose an alternative is to be found.

\section{Methods}

The designed algorithm is suitable for single channel data analysis, as well as analysis across multiple channels. In the first step the data is filtered by a digital Butterworth filter with a high pass frequency of $200 \mathrm{~Hz}$ and a low pass frequency of $3000 \mathrm{~Hz}$ [3]. These parameters were

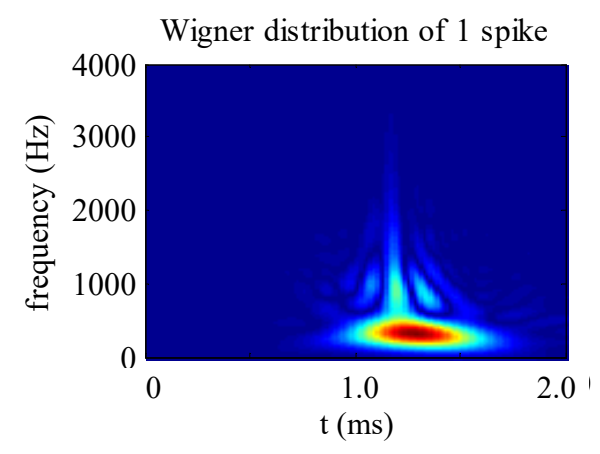

Figure 1: Time-frequency distribution of one neuronal spike extracted from a Wigner time-frequency distribution (Fig. 1).

The algorithm begins with the detection of all possible spikes which exceed a threshold. The threshold is based on a value which is calculated by the median multiplied with an empirically determined factor.

For spike sorting the maximum was fixed. The pre- and postsamples according to the maximum are used to determine a defined time course of the sequence [4].

Initially all defined spike sequences are collected associated to their channel. The first spike is chosen as form based reference. As well this spike forms the first element of the initial cluster. For each individual time course of a spike the correlation $(\mathrm{r}=0.6)$ to the reference spike is calculated. If the correlation factor between two spikes is above 0.6 , the spike is added to the current cluster. If the correlation is too low the spike is stored in a temporary container. After running through all the spikes and organize them, the container size is checked. In case of less than 30 unclassified spikes in the container, the algorithm stops otherwise a new cluster is opened. The first spike of the temporary container is chosen as reference for the new cluster and all other unclassified spikes will be checked for correlation.

\section{Results}

For validation the settings of the new spike sorting algorithm and the reference algorithm wave_clus are adjusted. The algorithm was tested with the same reference data which was also used by wave_clus. In Tab. 1 the results calculated by wave_clus and our new algorithm is shown.

Table 1: Number of classified spikes

\begin{tabular}{lll}
\hline cluster type & wave_clus & correlation \\
\hline cluster 1 & 182 & 182 \\
cluster 2 & 170 & 169 \\
cluster 3 & 167 & 169 \\
\hline
\end{tabular}

Applied to one channel simulated data, the algorithm delivers results which match mostly congruently with wave_clus. The number of detected spikes in wave_clus is $5 \overline{19}$, the correlation algorithm detects 520 . The maximum values of the same detected spikes vary between the two algorithms for \pm 5 samples. Both algorithms divide the founded spikes in three clusters (see Fig. 2). For better definition of a cluster all spikes will be averaged to one waveform (red, blue and green line in Fig. 2). 
Fig. 3 and Fig. 4 show an extension of the correlation algorithm to a multiple numbers of channels. The correlation was calculated over all channels.

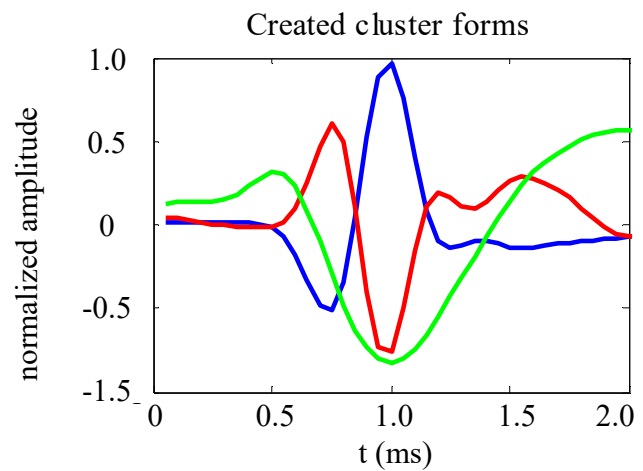

Figure 2: Cluster of single channel test data; average waveform of cluster 1 (blue), cluster 2 (red) and cluster 3 (green)

\section{Discussion and Outlook}

The comparability of the clusters is limited because the methodology of wave_clus changes the spike waveform in a severe way. Especially polyphasic spikes are no longer recognizable as such. An advantage compared to wave_clus is, that the arrays consisting of more channels can be considered in a causal relationship and the occurrence of certain spike shapes can be analyzed across all channels.

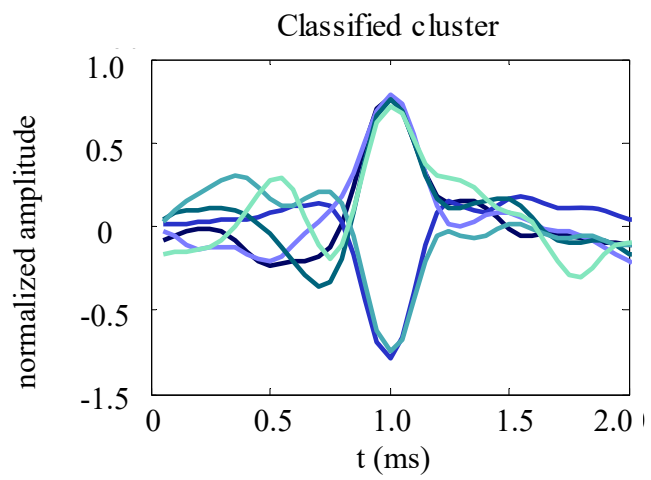

Figure 4: Mean of all classified spikes of the 9-channel MEA using correlation algorithm

In future work the algorithm will be used for MEAs with more than 48 channels. With these classified information a causality analysis could be calculated.

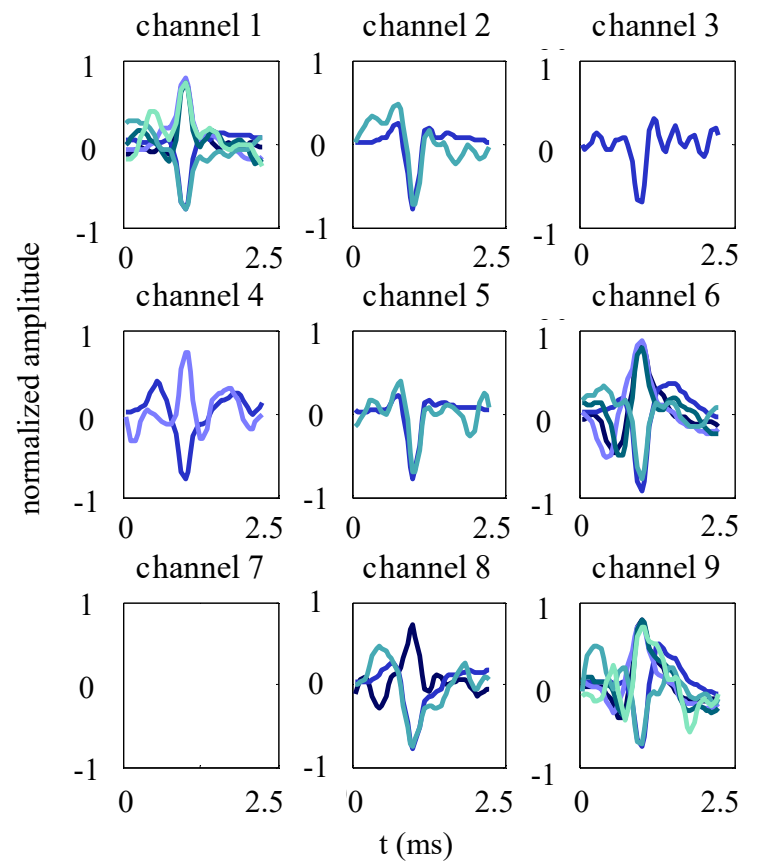

Figure 3: Mean of classified spikes per channel using a 9-channel MEA (no classified spike in channel 7)

\section{Bibliography}

[1] Klas H. Pettersen, Henrik Lindén, Anders M. Dale, and Gaute T. Einevoll, Extracellular spikes and currentsource density, Romain Brette, Alain Destexhe (Eds.), Handbook of Neural Activity Measurement, Cambridge University Press, 2012

[2] Quian Quiroga, R., Nadasdy, Z. et al.: Unsupervised Spike Detection and Sorting with Wavelets and Superparamagnetic Clustering, in Neural Computation $16,1661-1687,2004$

[3] K. Guillory und R. Normann. A 100 -channel system for real time detection and storage of extracellular spike waveforms. Journal of Neuroscience Methods, 91 (1-2):21-29, 1999.

[4] U. Rutishauser, E. M. Schuman und A. N. Mamelak. Online detection and sorting of extracellularly recorded action potentials in human medial temporal lobe recordings, in vivo. Journal of neuroscience methods, 154 (1-2):204-24, June 2006. 